\title{
Du défi naît l'occasion : La renaissance du phénix
}

\author{
par Richard Jones
}

L e titre de cet article reflète clairement le point de vue d'un optimiste. En effet, comment peut-on tirer quelque chose de positif de chaque défi? En bref, le sous-titre fait référence au phénix, oiseau mythique qui, brûlé, renaît de ses cendres. Dans le contexte moderne d'aujourd'hui, on pourrait faire le parallèle avec les revues d'affaires qui donnent en exemples depuis des années des personnes qui ont su saisir une occasion là où aucun n'en voyait. Ces personnes ont su se relever dans l'adversité. Dans le contexte de la récession économique actuelle, les gourous de la politique et de l'économie nous disent que nous avons atteints le fond du baril, mais en fait nous commençons à peine à ressentir les conséquences très difficiles sur les services de santé hospitaliers au Canada.

L'un des exemples de ces conséquences, les établissements de santé sont restructurés radicalement en systèmes très larges de type régie régionale, voire même en organisations à l'échelle d'une province, dans un effort de réduction des coûts de santé tout en s'assurant qu'un maximum de fonds publics soient utilisés à la prestation des soins de santé. Ces changements exigent donc des leaders dans les établissements de santé qu'ils acceptent davantage de responsabilités; or, cette situation a pour effet d'amener certains d'entre eux à réorienter leur carrière, voire à quitter carrément le milieu de la prestation des soins de santé. Néanmoins, de ce "lit de cendres ", les pharmaciens et d'autres professionnels de la santé saisissent l'occasion pour trouver de nouvelles avenues à la prestation des soins de santé, notamment la création de cliniques de soins primaires et de cliniques spécialisées, se rapprochant ainsi du patient sur tous les fronts. Cette transition vers la prestation des soins aux patients dans un nombre grandissant de milieux ambulatoires, même au sein des établissements de santé, voit un nombre croissant de pharmaciens exercer leur métier à côté des médecins et du personnel infirmier, à titre de membres essentiels de l'équipe soignante. Les leaders des établissements de santé trouvent des moyens pour s'assurer d'avoir l'infrastructure appropriée favorable à ces changements, mais le besoin de leaders munis de compétences comme nous les avons connus par le passé est aussi en mutation.
Les pharmaciens deviennent graduellement des praticiens autonomes et par conséquent des leaders de leur propre exercice professionnel dans un nombre croissant de milieux. Les leaders traditionnels ont souvent été difficiles à trouver, mais ils seront bientôt remplacés par des personnes possédant de nouvelles compétences, comme dans la gestion de système de logistique, de production ou de distribution, ou qui agiront comme des leaders-praticiens cliniciens, analogues aux leaders parmi nos collègues en médecine et en sciences infirmières. Les spécialités en pharmacie, la formation qui leur est nécessaire ainsi que la délivrance des titres correspondants constitueront les fondements du leadership en pratique de cette nouvelle catégorie de leaders. Les techniciens en pharmacie autorisés agiront à titre de leaders opérationnels de l'avenir, libérant ainsi entièrement les pharmaciens pour qu'ils deviennent des praticiens.

L'avènement de ces nouveaux leaders-pharmaciens cliniciens représentera une " renaissance des cendres " puisque leur partenariat avec les médecins et le personnel infirmier engendrera un nouveau modèle global de soins conjugués. Et imaginez : tout cela se produira dans le cadre d'une imposante récession économique et de la restructuration du système de soins de santé canadien qui en résulte. Beaucoup voient déjà les occasions qui se présentent et travaillent discrètement pour veiller à ce que la renaissance des cendres soit forte, considérable et durable. D'autres ne perçoivent pas encore les perspectives. Pour tirer pleinement parti des possibilités, nous devons découvrir les jeunes leaders parmi nos pairs des communautés pharmaceutique, médicale et infirmière et travailler en équipe avec eux pour offrir aux patients qui nous tiennent tant à cœur des services cliniques bien structurés, efficaces et de grande valeur.

[Traduction par l'éditeur]

Richard Jones, R. Ph., B. Sc., B.S.P., est président sortant et agent de liaison interne de la SCPH. 